BMJ Open Ophthalmology

\title{
Autofluorescence of choroidal vessels in Bietti's crystalline dystrophy
}

\author{
Hossein Ameri (1) , Erin Su, Tyler J Dowd-Schoeman
}

To cite: Ameri H, Su E, Dowd-Schoeman TJ. Autofluorescence of choroidal vessels in Bietti's crystalline dystrophy. BMJ Open Ophthalmology 2020;5:e000592. doi:10.1136/ bmjophth-2020-000592

Received 6 August 2020 Revised 10 October 2020 Accepted 10 October 2020
Check for updates

(c) Author(s) (or their employer(s)) 2020. Re-use permitted under CC BY-NC. No commercial re-use. See rights and permissions. Published by BMJ.

USC Roski Eye Institute, Department of Ophthalmology, Keck School of Medicine, University of Southern California, Los Angeles, California, USA

Correspondence to Dr Hossein Ameri; ameri@med. usc.edu

\section{ABSTRACT}

Objective To describe the pattern of fundus autofluorescence (FAF) in Bietti's crystalline dystrophy (BCD).

Methods and analysis From the National Institutes of Health EyeGene database of 2769 patients with known pathogenic mutations, 5 patients with BCD-causing CYP4V2 mutations who had FAF images were selected. Demographic and genetic information and imaging files were obtained. From the FAF imaging files, unique autofluorescence (AF) patterns and correlation with retinal structures were assessed by three investigators for clinical significance.

Results Five patients (four males, one female; mean age 56 years, range $42-76$ years) were included, all with different CYP4V2 mutations. All patients displayed varying degrees of hypo-AF in the posterior pole. In four out of five patients, there was a relative hyper-AF of choroidal vessels within the hypo-AF area; this feature was limited to sclerotic vessels only. A transitional zone of speckled AF was visible around the hypo-AF area. This zone corresponded to the area containing retinal crystals on colour fundus photography; however, retinal crystals did not demonstrate hyper or hypo-AF.

Conclusions This study presents a previously unreported characteristic finding in patients with $B C D$ with CYP4V2 mutations. AF of choroidal vessels may aid in differentiating BCD from other retinal dystrophies.

\section{INTRODUCTION}

Bietti's crystalline dystrophy (BCD) is a rare, progressive chorioretinal degenerative disease that affects an estimated 1/67 000 individuals, ${ }^{1}$ predominantly those of East Asian descent. ${ }^{2}$ Age of onset is typically in the third decade of life, with clinical manifestations of decreased visual acuity, night blindness and visual field loss, which may be central or peripheral. ${ }^{3}$ Ocular exam may reveal crystalline deposits in the cornea and/or retina and sclerosis of choroidal vessels. ${ }^{13}$ In latestage BCD, the intraretinal crystals disappear and give way to chorioretinal and retinal pigment epithelium (RPE) atrophy, ${ }^{4}$ and typically patients become legally blind by their 40-50s. ${ }^{1}$ BCD is inherited in an autosomal recessive pattern, and is due to mutations in the CYP4V2 gene. ${ }^{5}$ CYP4V2 is ubiquitously expressed in various tissues in the body, including the cornea and retina, and encodes

\section{Key messages}

What is already known about this subject?

Previous studies have reported abnormal fundus autofluorescence in Bietti's crystalline dystrophy.

\section{What are the new findings?}

$>$ Our study demonstrates autofluorescence of sclerotic choroidal vessels in Bietti's crystalline dystrophy, a distinct pattern that has not been previously reported.

\section{How might these results change the focus of research or clinical practice? \\ - The characteristic pattern of fundus autofluores- cence observed in this study is useful in clinically differentiating Bietti's crystalline dystrophy from other retinal dystrophies and can provide insight into the pathogenesis of the disease.}

for Cytochrome P450 4V2, an enzyme that is responsible for fatty acid metabolism. ${ }^{5}$ The hallmark crystalline inclusions of BCD have also been found outside of ocular tissue, including circulating lymphocytes and skin fibroblasts, suggesting that BCD is caused by a systemic dysfunction of lipid metabolism. ${ }^{36}$ However, there is extreme variability in BCD disease presentation. For example, progression may be asymmetric between the two eyes, ${ }^{7}$ and individuals with the same CYP4V2 mutation may present differently. ${ }^{89}$ As such, with its rarity, early asymptomatic stages, painless progression and high variability, BCD can be difficult to diagnose and study. Previous imaging modalities have been used to better characterise $\mathrm{BCD}$; these include fundus photography, near infrared reflectance, fluorescein angiography, fundus autofluorescence (FAF) and others.

FAF is an efficient, non-invasive clinical imaging modality that reflects RPE lipofuscin distribution, using a known wave length excitation to detect lipofuscin emissions. ${ }^{10}$ Lipofuscin naturally accumulates in the RPE over time as a byproduct of the incomplete degradation of photoreceptor outer segments. ${ }^{112}$ Though retinal lipofuscin accumulation is a hallmark of normal ageing, 
abnormal patterns in lipofuscin density indicate certain retinal pathologies, and can be assessed accordingly with FAF. Previous clinical uses of FAF most prominently include age-related macular degeneration (AMD), ${ }^{13} 14$ which features abnormal lipofuscin accumulation, ${ }^{15}$ as well as many other macular dystrophies, ${ }^{16}$ including Stargardt disease ${ }^{10}$ and retinitis pigmentosa. ${ }^{17}$ There are various $\mathrm{FAF}$ wavelengths used, short-wavelength versus near infrared; there are also different FAF imaging systems, including fundus camera, confocal scanning laser ophthalmoscopes and ultrawidefield technologies. ${ }^{18}$ Regardless of their different imaging strategies, all FAF technologies are able to act as metabolic mapping tools of the retina and assess RPE overall health or dysfunctions.

Previous studies have reported FAF changes in patients with BCD. This study presents autofluorescence (AF) changes in $\mathrm{BCD}$ with particular focus on previously undescribed AF findings in choroidal vessels.

\section{MATERIALS AND METHODS}

This is a retrospective study of the National Institutes of Health (NIH) EyeGene database. The deidentified data were obtained from the NIH EyeGene database, a DNA repository and patient registry of patients with inherited eye diseases from various institutions across the USA and Canada. Patients or the public were not involved in the design, or conduct, or reporting, or dissemination plans of this research. From 2769 patients with known pathogenic mutations, 10 patients with mutations in CYP4V2 causing BCD were selected. Five patients who did not have FAF images were excluded. Demographic information, genetic information and imaging files including colour fundus photo and FAF images of the five remaining patients were collected. FAF and colour images were then reviewed by three investigators for unique patterns of $\mathrm{AF}$ and their correlation with retinal structures. Although the information on the type of FAF images were not available, all images appear to be shortwavelength FAF; two images appear to have been taken with ultrawidefield Optos camera and three with Heidelberg Spectralis equipment.

\section{RESULTS}

The study included five patients (table 1 ), four men and one woman, with a mean age of 56 years (range 42-76 years). Four out of five patients were non-Hispanic white and one was black. Mean age onset of night blindness was 48 years (range 32-64 years). Mean duration of night blindness was 11 years (range 4-21 years). Snellen visual acuity ranged from 20/30 to 20/200 in three patients; for the remaining two patients, no visual acuity was available. Three out of five had bilateral corneal crystals. Four out of five patients had homozygous mutations, and one had compound heterozygous mutations, with no two patients sharing the same exact CYP4V2 mutation (table 1).

On evaluation of FAF images, posterior pole hypo-AF was observed in all patients, reflecting RPE atrophy (figure 1). In all but one patient (patient 5), the hypo-AF area was contiguous and involved the macula and peripapillary region. In these patients, choroidal vasculature was visible on FAF and demonstrated a relative hyper-AF compared with the surrounding hypo-AF area. Colour fundus photos were available for three patients (patients 2, 4 and 5), making it possible to assess for the presence of choroidal sclerosis and retinal crystals in those. In patient 2, all vessels were sclerotic and all showed relative hyper-AF. In patient 5, there was no choroidal sclerosis, and choroidal vessels were not visible on FAF; this patient only had geographical patches of hypo-AF, indicating less advanced disease compared with the others (figure 2). In patient 4 , there was a mixture of sclerotic and nonsclerotic vessels, and only sclerotic vessels showed AF (figure 3). Patients 2, 4 and 5 showed a transitional zone of speckled AF surrounding the hypo-AF area; all of them displayed retinal crystals on colour fundus photos which corresponded to the speckled AF zone. However, retinal crystals did not consistently show hyper or hypo-AF (figure 2). Patients 1 and 3 showed irregularly shaped islands of relative hyper-AF within the hypo-AF area in a pattern somewhat similar to that in choroideraemia (figure 1A,C).

\section{DISCUSSION}

Retinal pathologies, like BCD, present different patterns of hyper-AF and hypo-AF. Previous studies have reported FAF patterns in BCD, including diffuse or multilobular

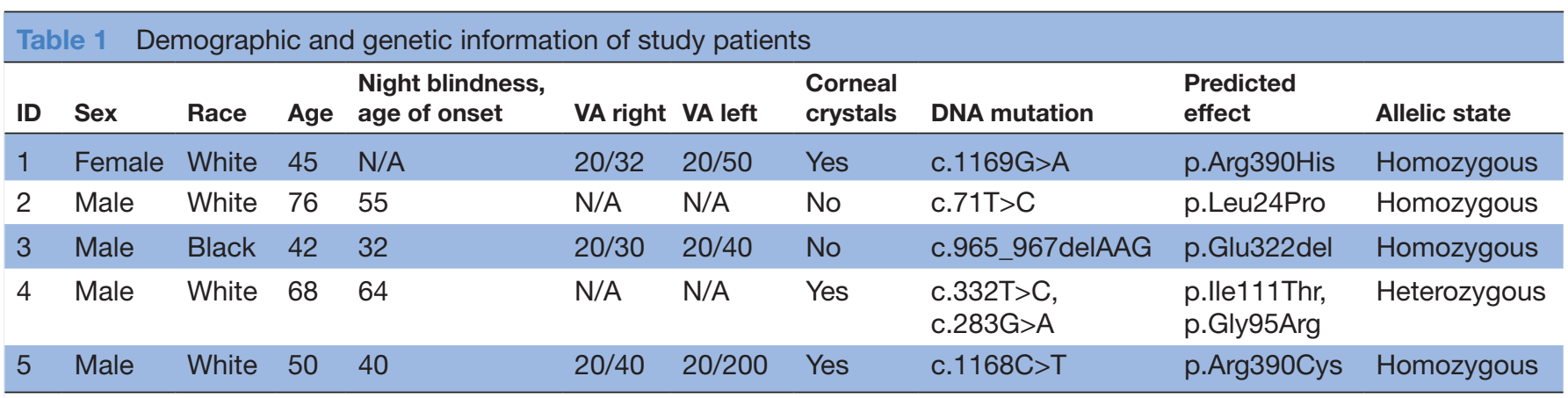

N/A, not available; VA, visual acuity. 


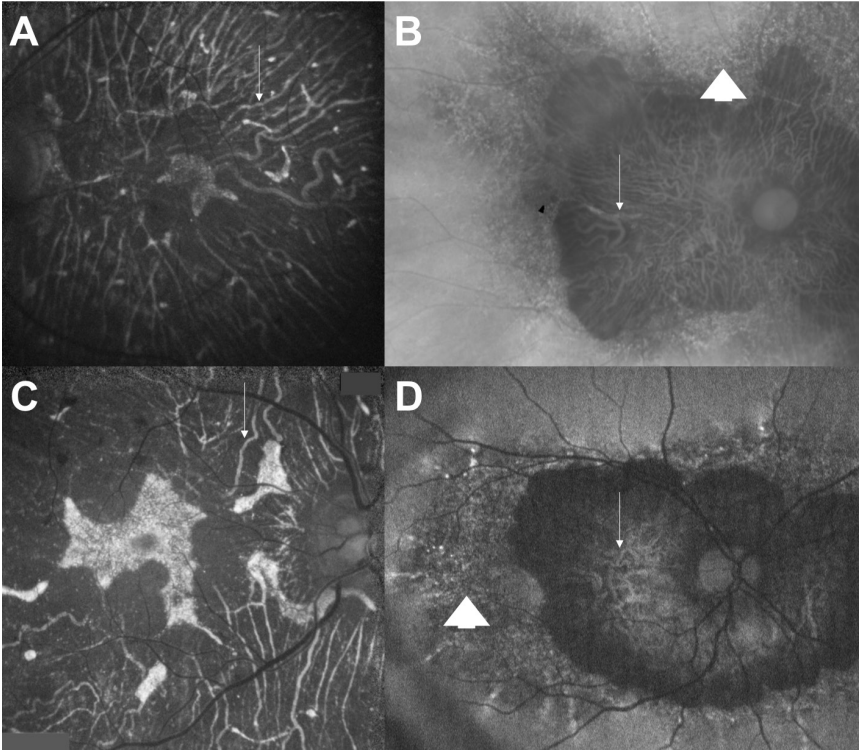

Figure 1 Fundus autofluorescence images of four patients with diffuse hypo autofluorescence (AF) of posterior pole involving the macula and peripapillary area. Choroidal vessels (arrows) show AF in all four patients. (A) Patient 1, left eye, an island of relative hyper-AF is visible within the diffuse hypo-AF area. (B) Patient 2, right eye, a transitional zone with speckled AF (arrowhead) surrounds the hypo-AF area. (C) Patient 3, right eye, islands of relative hyper-AF are visible within the diffuse hypo-AF area. (D) Patient 4, right eye, a transitional zone with speckled AF (arrowhead) surrounds the hypo-AF area.

hypo-AF of posterior pole, and hyper-AF and hypo-AF spots. ${ }^{19-21}$ An interesting finding in our study is AF of choroidal vasculature, which has not been previously reported. Fuerst $e t a l^{20}$ reported a patient with BCD with choroidal neovascularisation, and described 'tenuously hyper-AF' dots overlying the choroidal vessels seen on near-infrared FAF images in the contralateral eye. They

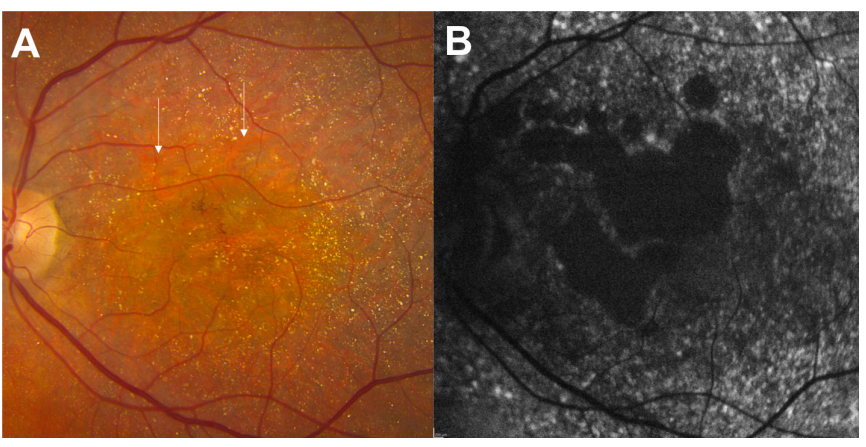

Figure 2 Images of the left eye of patient 5. (A) Colour fundus photo shows numerous retinal crystals appearing as tiny yellow dots. Retinal pigment epithelium atrophy is not obvious, but choroidal vessels (arrows) can be seen without any sign of choroidal sclerosis. (B) Fundus autofluorescence image displays geographical areas of hypo autofluorescence (AF) centrally surrounded by diffuse speckled AF. Note lack of AF of choroidal vessels. Also, hyper- or hypo-AF spots essentially do not correspond to retinal crystals on the colour image.

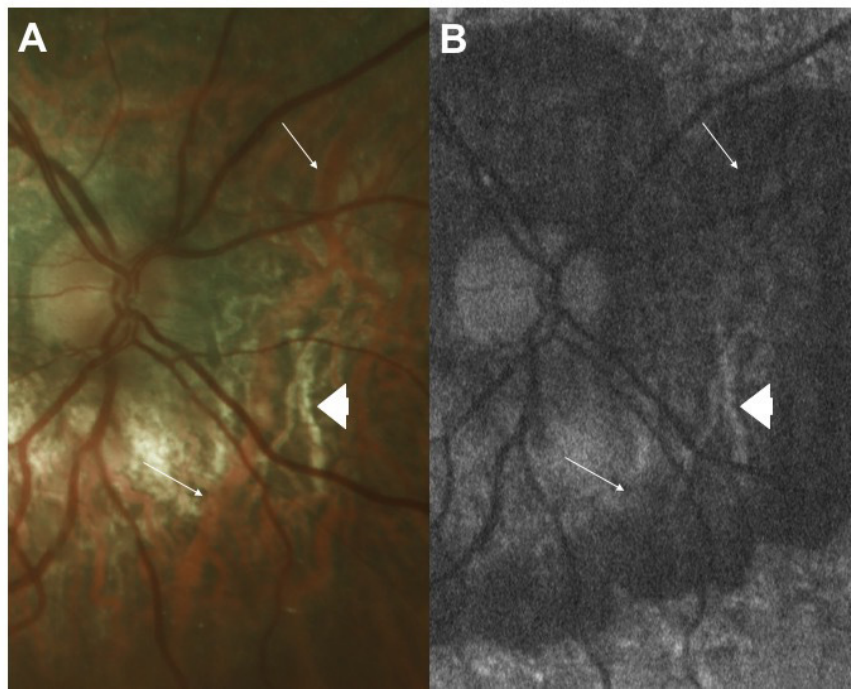

Figure 3 Images of the nasal retina of the right eye of patient 4. (A) Colour fundus photo shows both sclerotic (arrowhead) and non-sclerotic (arrow) choroidal vessels. (B) Fundus autofluorescence image shows autofluorescence (AF) of sclerotic choroidal vessels (arrowhead); however, nonsclerotic vessels do not display AF, and largely are not visible expect superiorly that show a faint hypo-AF (arrows point to the location of non-sclerotic choroidal vessels based on the colour fundus photo).

also described mottled hypo-AF of the macula on shortwavelength FAF. A closer look at their FAF pictures, speckled AF with predominance of hypo-AF spots can be seen on short-wavelength FAF without any visibility of choroidal vessels. However, choroidal vessels are visible on the near-infrared FAF image and primarily display hypo-AF. Li et $a l^{19}$ and Oishi $e t a l^{21}$ have also reported AF changes in BCD but neither have commented on AF of choroidal vasculature; however, on two of the patients presented in the Oishi et $a l^{21}$ paper, AF of choroidal vasculature is evident on FAF images.

Despite the different FAF imaging systems and calibrations used to study patients, our FAF observations remain largely consistent between our patients, with FAF showing $\mathrm{AF}$ of choroidal vessels in four of five patients, giving the appearance of relative hyper-AF in the surrounding hypo-AF area. The only patient who did not show this feature was the one who had smaller area of RPE atrophy and no choroidal sclerosis on colour fundus photo, suggesting less advanced disease.

As to what causes AF of choroidal vessels is unclear. Choroidal sclerosis is one of the distinguished features of BCD, and this study shows that choroidal vascular AF is primarily seen when there is sclerosis. Presence of fibrotic changes in the choroid has been reported in BCD. ${ }^{3}$ Additionally, lipid materials, which often contain liposomal crystals, have been reported in conjunctival fibroblasts, circulating lymphocytes and choroidal fibroblasts of patients with BCD. ${ }^{36223}$ The crystals themselves are less likely to be the source of AF, as retinal crystals do not display AF on short-wavelength FAF; lipid granules, 
however, may contain fluorophores and show AF. We hypothesise that AF of choroidal vasculature in BCD is caused by fibroblasts and fibrotic materials around the large choroidal vessels which contain fluorophores, likely within the lipid granules. Detailed ultrastructural studies of vessel wall in patients with BCD with choroidal sclerosis may help to discover the source of AF and further expand our knowledge on the pathogenesis of the disease. Choroidal vessel sclerosis may also be seen in chroideraemia, which has some phonotypical resemblance to $\mathrm{BCD} .^{24}$ Zhang et $a l^{24}$ reported significant plasma fatty acid abnormalities and prominent crystals in circulating lymphocytes suggesting presence of systemic disease in choroideremia similar to BCD. In our study, we observed islands of relative hyper-AF within the central hypo-AF area, which is typically seen in choroideraemia. A study on the frequency of hyper-AF of choroidal vessels in choroideraemia and its potential association with choroidal sclerosis may further shed light on the pathogenesis of both diseases.

AF of choroidal vessels is not limited to BCD. Shinojima $e t a l^{25}$ reported hyper-AF of choroidal vessels within the macular atrophic area in over half of eyes with dry AMD and about $16 \%$ of eyes with macular dystrophy. The type of macular dystrophy was not specified and it is not known whether any of those patients had BCD. Their study found that choroidal thickness was significantly less in eyes that showed hyper-AF of choroidal vessels. Future large studies on BCD may explore any potential association between choroidal thickness and AF of choroidal vessels.

A common feature in our patients was hypo-AF of the posterior pole, suggesting diffuse loss of RPE and hence advanced disease. Additionally, we observed a transitional zone of speckled AF surrounding the hypo-AF area in those with wide field images. This finding is similar to the case presented by Fuerst $e t a l^{20}$ except that their case did not show a central area of hypo-AF at the posterior pole. We also noted the lack of any specific appearance of retinal crystalline deposits on FAF, which corroborates with the findings reported by Oishi $e t a l^{21}$

There are some limitations to this study. First, the image bank was limited and each patient had one modality of FAF images, which varied between patients. However, this had some advantages in that we were able to demonstrate similar changes on different FAF modalities. Second, the study was retrospective, and we could not exactly determine the stage of the disease and establish at what point choroidal vessels started showing AF. Nevertheless, the display of large areas of hypo-AF at the posterior pole of most images indicates significant RPE loss and relatively advanced disease in the majority of patients. Third, this study included a small number of patients; larger studies are needed to show the frequency of our findings in patients with BCD.

In conclusion, this study demonstrates that, despite the inherent genotypic and phenotypic variability of $\mathrm{BCD}$, FAF characteristics of BCD remain largely consistent.
Relative hyper-AF of sclerotic choroidal vessels within the hypo-AF area of the posterior pole may aid in more affirmative clinical diagnosis of $\mathrm{BCD}$ in the absence of genetic testing or when genetic testing is inconclusive; particularly it can be useful in differentiating BCD from other crystalline retinopathies.

Acknowledgements We would like to thank the eyeGENE participants and the eyeGENE Research Group for their valuable contribution to this research.

Contributors HA: inception, data analysis and manuscript writing. ES: data analysis and manuscript writing. TJD-S: data collection, data analysis and manuscript revision

Funding The data used for the analyses described in this paper were obtained from the National Eye Institute - National Ophthalmic Genotyping and Phenotyping Network (eyeGENE - Protocol 06-El-0236 which has been funded in part from the National Institutes of Health/National Eye Institute, under Contract No. HHS-N-2602007-00001-C. Supported in part by an Unrestricted Grant to the Department of Ophthalmology from Research to Prevent Blindness, New York, NY.

\section{Competing interests None declared.}

Patient and public involvement Patients and/or the public were not involved in the design, or conduct, or reporting, or dissemination plans of this research.

Patient consent for publication Not required.

Ethics approval Institutional Review Board approval was obtained from the Office for the Protection of the Research Subjects of the University of Southern California (protocol number: HS-19-00040).

Provenance and peer review Not commissioned; internally peer reviewed.

Data availability statement All data relevant to the study are included in the article.

Open access This is an open access article distributed in accordance with the Creative Commons Attribution Non Commercial (CC BY-NC 4.0) license, which permits others to distribute, remix, adapt, build upon this work non-commercially, and license their derivative works on different terms, provided the original work is properly cited, appropriate credit is given, any changes made indicated, and the use is non-commercial. See: http://creativecommons.org/licenses/by-nc/4.0/.

ORCID iD

Hossein Ameri http://orcid.org/0000-0002-5270-2800

\section{REFERENCES}

1 Vargas M, Mitchell A, Yang P, et al. Bietti crystalline dystrophy. In: Adam MP, ed. GeneReviews((R)). Seattle, WA, 1993.

$2 \mathrm{Hu}$ DN. Prevalence and mode of inheritance of major genetic eye diseases in China. J Med Genet 1987;24:584-8.

3 Kaiser-Kupfer MI, Chan CC, Markello TC, et al. Clinical biochemical and pathologic correlations in Bietti's crystalline dystrophy. Am J Ophthalmol 1994;118:569-82.

4 Mansour AM, Uwaydat SH, Chan C-C. Long-term follow-up in Bietti crystalline dystrophy. Eur J Ophthalmol 2007;17:680-2.

$5 \mathrm{Li} \mathrm{A}$, Jiao X, Munier FL, et al. Bietti crystalline corneoretinal dystrophy is caused by mutations in the novel gene CYP4V2. Am J Hum Genet 2004;74:817-26.

6 Wilson DJ, Weleber RG, Klein ML, et al. Bietti's crystalline dystrophy. A clinicopathologic correlative study. Arch Ophthalmol 1989;107:213-21.

7 García-García GP, Martínez-Rubio M, Moya-Moya M-A, et al. Identification of novel CYP4V2 genotypes associated with Bietti crystalline dystrophy and atypical anterior segment phenotypes in Spanish patients. Acta Ophthalmol 2018;96:e865-73.

8 Fong AMY, Koh A, Lee K, et al. Bietti's crystalline dystrophy in Asians: clinical, angiographic and electrophysiological characteristics. Int Ophthalmol 2009;29:459-70.

9 Haddad NMN, Waked N, Bejjani R, et al. Clinical and molecular findings in three Lebanese families with Bietti crystalline dystrophy: report on a novel mutation. Mol Vis 2012;18:1182-8.

10 Delori FC, Staurenghi G, Arend O, et al. In vivo measurement of lipofuscin in Stargardt's disease--Fundus flavimaculatus. Invest Ophthalmol Vis Sci 1995;36:2327-31.

11 Wing GL, Blanchard GC, Weiter JJ. The topography and age relationship of lipofuscin concentration in the retinal pigment epithelium. Invest Ophthalmol Vis Sci 1978;17:601-7. 
12 Boulton M, McKechnie NM, Breda J, et al. The formation of autofluorescent granules in cultured human RPE. Invest Ophthalmol Vis Sci 1989;30:82-9.

13 von Rückmann A, Fitzke FW, Bird AC. Fundus autofluorescence in age-related macular disease imaged with a laser scanning ophthalmoscope. Invest Ophthalmol Vis Sci 1997;38:478-86.

14 Delori FC, Fleckner MR, Goger DG, et al. Autofluorescence distribution associated with drusen in age-related macular degeneration. Invest Ophthalmol Vis Sci 2000;41:496-504.

15 Dorey CK, Wu G, Ebenstein D, et al. Cell loss in the aging retina. Relationship to lipofuscin accumulation and macular degeneration. Invest Ophthalmol Vis Sci 1989;30:1691-9.

16 von Rückmann A, Fitzke FW, Bird AC. In vivo fundus autofluorescence in macular dystrophies. Arch Ophthalmol 1997;115:609-15.

17 von Rückmann A, Fitzke FW, Bird AC. Distribution of pigment epithelium autofluorescence in retinal disease state recorded in vivo and its change over time. Graefes Arch Clin Exp Ophthalmol 1999;237:1-9.

18 Yung M, Klufas MA, Sarraf D. Clinical applications of fundus autofluorescence in retinal disease. Int J Retina Vitreous 2016;2:12.

19 Li Q, Li Y, Zhang X, et al. Utilization of fundus autofluorescence, spectral domain optical coherence tomography, and enhanced depth imaging in the characterization of Bietti crystalline dystrophy in different stages. Retina 2015;35:2074-84.

20 Fuerst NM, Serrano L, Han G, et al. Detailed functional and structural phenotype of Bietti crystalline dystrophy associated with mutations in CYP4V2 complicated by choroidal neovascularization. Ophthalmic Genet 2016;37:445-52.

21 Oishi A, Oishi M, Miyata M, et al. Multimodal imaging for differential diagnosis of Bietti crystalline dystrophy. Ophthalmol Retina 2018;2:1071-7.

22 Welch RB. Bietti's tapetoretinal degeneration with marginal corneal dystrophy crystalline retinopathy. Trans Am Ophthalmol Soc 1977;75:164-79.

23 Furusato E, Cameron JD, Chan C-C. Evolution of cellular inclusions in Bietti's crystalline dystrophy. Ophthalmol Eye Dis 2010;2010:9-15.

24 Zhang AY, Mysore N, Vali $\mathrm{H}$, et al. Choroideremia is a systemic disease with lymphocyte crystals and plasma lipid and RBC membrane abnormalities. Invest Ophthalmol Vis Sci 2015;56:8158-65.

25 Shinojima A, Mori R, Nakashizuka $\mathrm{H}$, et al. Characteristics of Hyperautofluorescent choroidal vessels within the macular atrophic area using spectral-domain optical coherence tomography. Ophthalmologica 2016;235:208-14. 